\title{
Assessment of Contamination of Underground Water Sources in Enyigba Pb-Zn District, South Eastern Nigeria using Metal Enrichment and Pollution Indices
}

\author{
Nnabo Paulinus N1
}

${ }^{1}$ Department of Geology, Ebonyi State University, Abakaliki, Nigeria

\begin{abstract}
Samples were collected from four (4) underground water sources in Enyigba Pb-Zn mining district, near Abakaliki in southeastern Nigeria. The samples were acidified to stabilize the metals for periods more than four days without the use of refrigeration. The acidified water samples were analysed by a commercial laboratory at Projects Development Institute (PRODA), Enugu using Atomic Absorption Spectroscopy (AAS). The elements determined by this method are lead $(\mathrm{Pb})$, zinc $(\mathrm{Zn})$, copper $(\mathrm{Cu})$, arsenic $(\mathrm{As})$, cadmium $(\mathrm{Cd})$, nickel $(\mathrm{Ni})$, manganese $(\mathrm{Mn})$ and cobalt $(\mathrm{Co})$. The result and analysis showed that metal enrichment index (MPI) of $\mathrm{As}, \mathrm{Cd}, \mathrm{Pb}$ and $\mathrm{Ni}$ are very high in the groundwater samples, indicating high degree of contamination compared to WHO permissible limits. All these metals contributed to high degree of contamination based on metal pollution index (MPI). The calculated metal enrichment and pollution indices show values that indicate that under the prevailing conditions and environmental regulations in Nigeria, the Enyigba mining district would face major and hazardous discharges of these metals to other water sources in the area.
\end{abstract}

Keywords: Metal Enrichment Index, Metal Pollution Index, Underground Water, Lead/Zinc Mines, Enyigba

\section{INTRODUCTION}

The study area (Figure 1), $14 \mathrm{~km}$ southeast of Abakaliki, covered the $\mathrm{Pb}-\mathrm{Zn}$ mining district of Enyigba and its surrounding villages of Ameka, Ameri and Ohankwu all in Ebonyi State, southeastern Nigeria. The deposit of $\mathrm{Pb}-\mathrm{Zn}$ sulphides (galena and sphalerite) in Nigeria have been known for a long time but have only been exploited in the past on a very small scale. The lead-zinc field covers over $48,000 \mathrm{sq} \mathrm{km}$ in extent with lead-zinc mineralization at many centres. The deposits are localized in the Cretaceous sediments along $600 \mathrm{~km}$ long belt within the Benue Trough, a sediment filled intracratonic basin extending from Ishiagu (South of Abakaliki) north-eastwards to Gombe (Farrington 1952, Orazulike 1994). The occurrence of lead-zinc in the Benue valley has attracted a lot of attention. Mining of the ore has been carried out for a long time by both the natives, for local uses as cosmetics, and foreign companies, for export. Production of the ore started in the year 1925 (Offodile, 1989) but commercial production started in 1947 (Kogbe, 1989). Since the discovery of and mining of $\mathrm{Pb}-\mathrm{Zn}$ deposits in Abakaliki and its environs in the early 1900s, data existing on the impact of their mining on the environment are very rare.

\begin{abstract}
Metal contamination that occurs as a result of mining characterized by elevated toxic metal concentrations and acid rock and mine drainage, continue several years after the cessation of mining activities. Heavy metal effluents from the weathering of the mineral deposits and mine dumps affect both the surface and underground water quality and soil (Igwe et al. 2012; Onyeobi and Imeokparia, 2014). These levels of contamination in the area lead to low agricultural production, and adversely affect the ecosystem if present at anomalously high level. This study is to assess the levels of heavy metals contamination of the surface and underground water as may have resulted from the lead-zinc mining in the area.
\end{abstract}

This article is published under the terms of the Creative Commons Attribution License 4.0

Author(s) retain the copyright of this article. Publication rights with Alkhaer Publications.

Published at: http://www.ijsciences.com/pub/issue/2015-09/

DOI: 10.18483/ijSci.825; Online ISSN: 2305-3925; Print ISSN: 2410-4477 


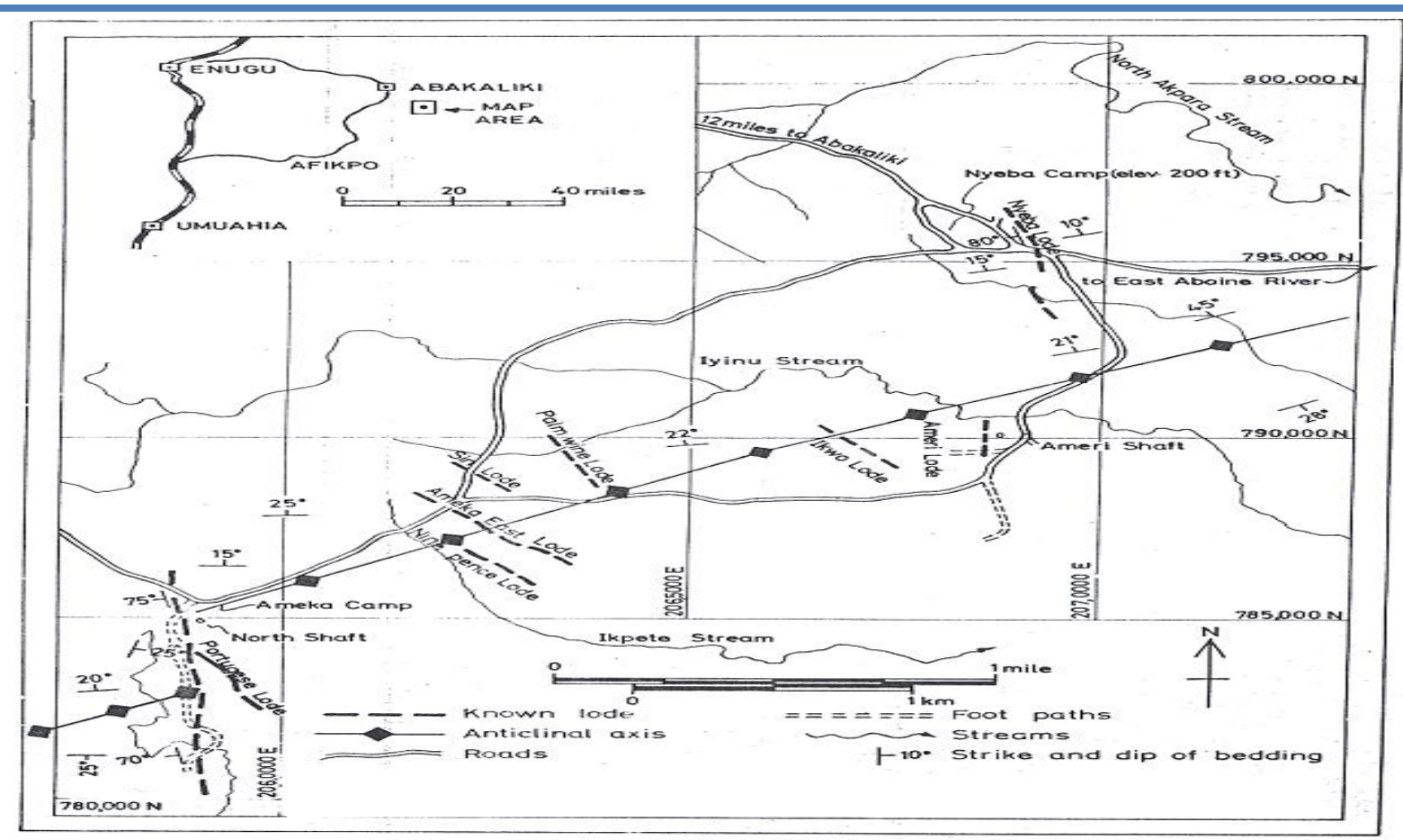

Figure 1 Geological map of lead-zinc deposits of Enyigba district, near Abakaliki, Lower Benue Trough. The area is underlain by Abakaliki shales (Modified from Orajaka, 1965).

\section{2: THE STUDY AREA}

In the study area, the topography is undulating plain alternating with running of ridges and hills from east to west. The plains are underlain by shale and some mudstones. The Enyigba, Ameri and Ameka are marked by undulating range of shale outcrops, which serve as the host for $\mathrm{Pb}-\mathrm{Zn}$ mineral ore bodies. The whole area formed the "Abakaliki anticlinorium" and generally underlain by shales. The area had about $60 \mathrm{~m}$ as its highest elevation and $30 \mathrm{~m}$ as its lowest elevation above sea level. The area falls within the tropical rainforest belt of South East Nigeria, and characterized by an average rainfall of 1750-2000 $\mathrm{mm}$ per annum. The highlands are characterized by drought resistance grasses, along stream and rivers. Among the vegetation includes economic mangoes trees, orange trees, and palm and coconut trees. The drainage system of the area is dendritic in pattern, which is a function of the lithology. The area is majorly drained by Ebonyi River. All the drainage systems flow eastward to join the Cross River somewhere outside the area.

The Abakaliki shale of lower Cretaceous age is exposed in the area. The sedimentary rocks are predominantly black calcareous (calcite-cemented) shale with occasional intercalation of siltstone (Figure 2). The shale formation belongs to the AsuRiver Group of the Albian Cretaceous sediments. The Asu River group which consists of alternating sequence of shales, mudstone and siltstone with some occurrence of sandstone and limestone lenses in some places and attains an estimated thickness of 1500 meters (Agumanu 1989, Farrington, 1952). Kogbe (1989) described the sediments as consisting of rather poorly-bedded sandy limestone lenses. The shales in some places are highly weathered and ferrugenized. The rocks are extensively fractured folded and faulted. From field observations, the rocks of the area consist of variably coloured shale and mudstone that has been imbedded by lead - zinc vein mineralization, baked intrusive shale as well as ironstone along veins. The ironstone occur as interbeds within the shale and as vein filling. The vein mineralization is hosted within the dark shale (Nnabo, 2011; Nnabo, 2015; Nnabo et al. 2011). The geology and mineral resources are the major factors responsible for availability of the heavy metals in the area. While the sulphide mineralization have high concentration of these metals, the shale host are capable of retaining them from ancient sea (Nnabo, 2011; Nnabo et al. 2011). 


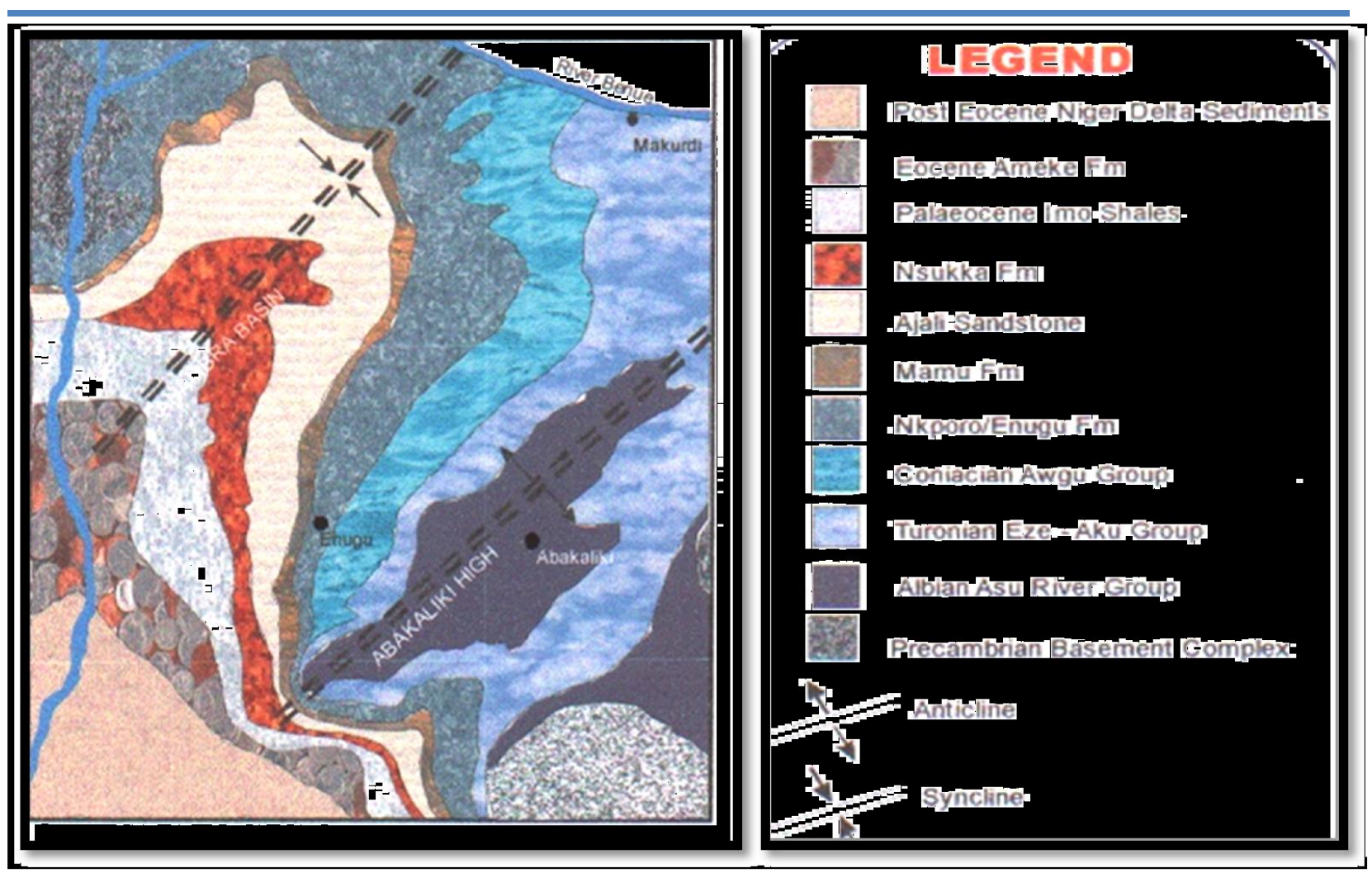

Figure 2 General geologic map of Southeastern Nigeria showing Abakaliki basin in the Lower Benue Trough (Modified after Hoque, 1977).

\section{METHODOLOGY}

Water sampling was carried out in the months of August and September. Sample locations (Figure 3) were located on 1:90,000 scale topographic map and recorded on site with a conventional global positioning system (GPS) instrument. Water samples for analyses were collected from surface (stagnant) and ground sources including mine ponds, mine pits, seeping from mine dumps, boreholes, and streams found in and around the area of mining activities. Notes were taken on the nature of any mineral coatings related to the water, if any, because these are often indicators of degraded water quality. Water samples were collected from sources where the water was deemed to be representative of a geological or mine setting and would yield information on the mobility of metals in that environment. Flow rate (for moving sources) and water characteristics (colour, suspended material, bed colours or mineralogy) are recorded and $\mathrm{pH}$ was measured using portable $\mathrm{pH}$ metre- HANNA pHep Hi 98127. The pH measurement is as plotted on Figure 4. The $\mathrm{pH}$ meter
(HANNA pHep Hi 98127) with built-in temperature electrode was a calibrated based on standards solution. The field standards were buffered solutions of $\mathrm{pH} 4.0,7.0$ and 10.0. The measurement of $\mathrm{pH}$ carries an uncertainty of about \pm 0.05 standard unit. The water sample for analyses was collected with disposable $60 \mathrm{ml}$ bottles. The disposable polyethylene bottle was rinsed three times in the sampled water prior to collection. The sample was acidified at the site with three drops of ultrapure 1:1 $\mathrm{HNO}_{3}$.

The acid stabilized metals for periods more than four days without the use of refrigeration. Laboratory and field blank tests were carried out using deionized water. The acidified water samples were analysed by a commercial laboratory at Projects Development Institute (PRODA), Enugu using Atomic Absorption Spectroscopy (AAS). The elements determined by this method are lead $(\mathrm{Pb})$, zinc $(\mathrm{Zn})$, copper $(\mathrm{Cu})$, arsenic (As), cadmium (Cd), nickel $(\mathrm{Ni})$, manganese $(\mathrm{Mn})$ and cobalt $(\mathrm{Co})$. 
Assessment of Contamination of Underground Water Sources in Enyigba Pb-Zn District, South Eastern Nigeria using Metal Enrichment and Pollution Indices

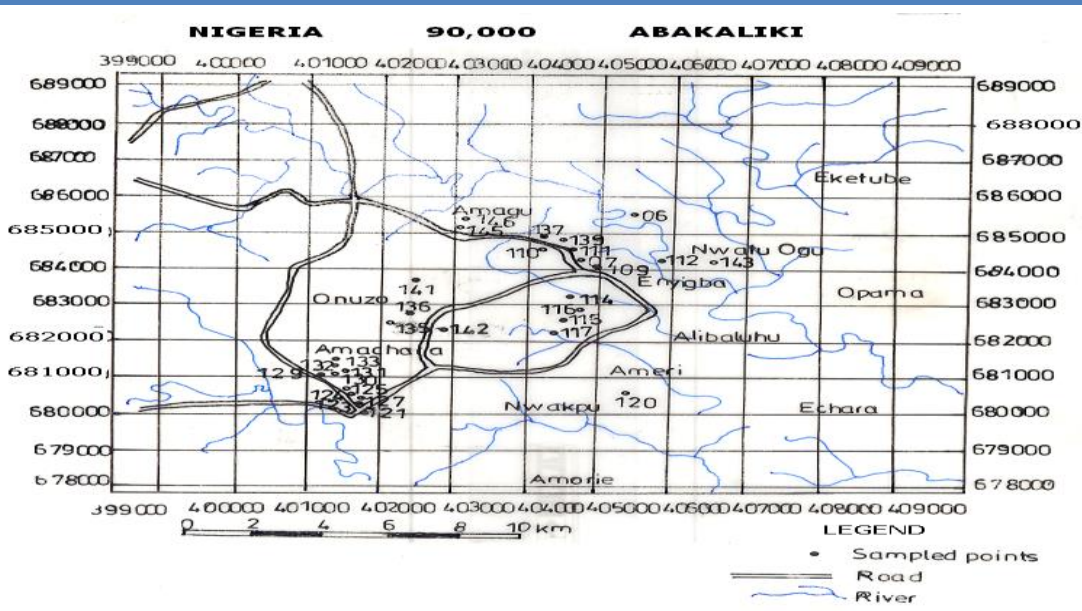

Figure 3 Location of Water Samples collected from Enyigba and its Environs

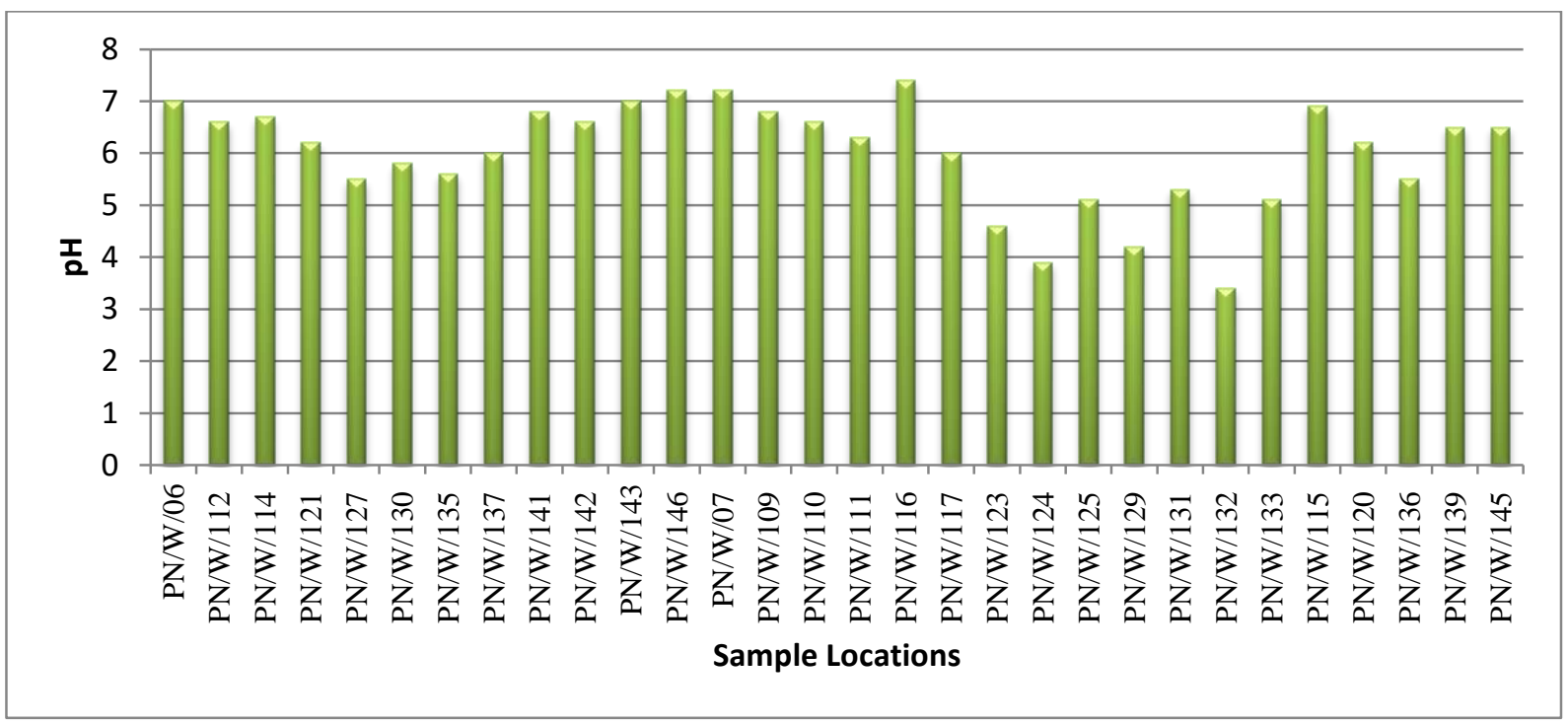

Figure 4 The plot of field measurement of $\mathrm{pH}$ of the water samples from Enyigba mining district

\section{RESULTSS OF HEAVY METAL ANALYSIS IN WATER.}

The results of the analysis of water samples for their contamination by heavy metals are shown in Tables 1 to 4 . The tables also contain the statistical data for each metal. The concentrations of each metal that are above the World Health Organization (WHO, 2011) established maximum limits of heavy metals permissible in water (Table 5) are highlighted.. The content of $\mathrm{As}, \mathrm{Cd}, \mathrm{Ni}$ and $\mathrm{Pb}$ in many of the samples are above the WHO maximum permissible limits for drinking water. The distributions of $\mathrm{As}, \mathrm{Cd}$ and $\mathrm{Pb}$ in all the sampled sites are plotted in Figures 5 to 7.

Table 1 Concentration of heavy metals in water (in $\mathrm{mg} / \mathrm{l}$ ) and its assessment with the maximum limits established by the World Health Organization (WHO, 2011). (NL = No limit).

\begin{tabular}{|l|l|l|l|l|l|l|l|l|l|l|l|}
\hline $\begin{array}{l}\text { Sample } \\
\text { No }\end{array}$ & Location & $\begin{array}{l}\text { Sample } \\
\text { type }\end{array}$ & $\mathbf{p H}$ & $\mathbf{A s}$ & $\mathbf{C d}$ & $\mathbf{C o}$ & $\mathbf{C u}$ & $\mathbf{M n}$ & $\mathbf{N i}$ & $\mathbf{P b}$ & $\mathbf{Z n}$ \\
\hline PN/W/06 & $\begin{array}{l}\text { Enyigba } \\
\text { Stream 1 }\end{array}$ & Surface & 7.0 & Nil & $\mathbf{3 . 2}$ & 4.2 & 0.17 & $\mathbf{0 . 4 2}$ & $\mathbf{1 . 4 9}$ & $\mathbf{0 . 7}$ & 0.56 \\
\hline PN/W/112 & $\begin{array}{l}\text { Enyigba } \\
\text { stream 2 }\end{array}$ & Surface & 6.6 & $\mathbf{0 . 3}$ & $\mathbf{6 . 0}$ & 1.26 & 0.08 & 0.06 & $\mathbf{1 . 4 3}$ & Nil & 0.54 \\
\hline PN/W/114 & Ameri stream & Surface & 6.7 & Nil & $\mathbf{1 . 4 0}$ & 1.71 & 0.06 & 0.11 & Nil & $\mathbf{0 . 2 0}$ & 0.09 \\
\hline PN/W/121 & $\begin{array}{l}\text { Ohankwu } \\
\text { stream 1 }\end{array}$ & Surface & 6.2 & Nil & $\mathbf{1 . 3 0}$ & 0.60 & 0.05 & 0.02 & $\mathbf{0 . 8 8}$ & $\mathbf{0 . 2 0}$ & 0.01 \\
\hline
\end{tabular}


Assessment of Contamination of Underground Water Sources in Enyigba Pb-Zn District, South Eastern Nigeria using Metal Enrichment and Pollution Indices

\begin{tabular}{|c|c|c|c|c|c|c|c|c|c|c|c|}
\hline PN/W/127 & $\begin{array}{l}\text { Ohankwu } \\
\text { stream } 2\end{array}$ & Surface & 5.5 & 2.50 & 13.5 & Nil & 0.02 & 0.21 & 0.97 & Nil & Nil \\
\hline $\mathrm{PN} / \mathrm{W} / 130$ & $\begin{array}{l}\text { Ameka } \\
\text { stream } 1\end{array}$ & Surface & 5.8 & 0.20 & 12.3 & Nil & Nil & 0.07 & 0.42 & 0.30 & 0.15 \\
\hline PN/W/135 & $\begin{array}{l}\text { Ameka } \\
\text { stream 2 }\end{array}$ & Surface & 5.6 & Nil & Nil & 0.97 & 0.02 & 0.04 & Nil & 0.20 & 0.27 \\
\hline PN/W/137 & $\begin{array}{l}\text { Enyigba villa } \\
\text { pond }\end{array}$ & Surface & 6.0 & 0.30 & Nil & 4.60 & 0.06 & 0.20 & 2.07 & 0.20 & Nil \\
\hline PN/W/141 & $\begin{array}{l}\text { Enyigba villa } \\
\text { stream } 1\end{array}$ & Surface & 6.8 & 0.20 & Nil & 2.39 & 0.03 & 0.26 & 1.58 & 0.10 & 9.70 \\
\hline $\mathrm{PN} / \mathrm{W} / 142$ & $\begin{array}{l}\text { Ameka villa } \\
\text { stream }\end{array}$ & Surface & 6.6 & 0.25 & Nil & 5.06 & 0.08 & 0.01 & 0.52 & 0.20 & 6.40 \\
\hline PN/W/143 & $\begin{array}{l}\text { Ebonyi River, } \\
\text { Enyigba }\end{array}$ & Surface & 7.0 & 0.65 & Nil & 3.90 & 0.04 & 0.197 & Nil & 0.10 & 10.1 \\
\hline PN/W/146 & $\begin{array}{l}\text { Enyigba villa } \\
\text { stream } 2\end{array}$ & Surface & 7.2 & 0.13 & Nil & 1.0 & 0.01 & 0.07 & 0.63 & 0.10 & 7.50 \\
\hline $\mathrm{PN} / \mathrm{W} / 07$ & $\begin{array}{l}\text { Enyigba Shale } \\
\text { quarry pond }\end{array}$ & Surface & 7.2 & Nil & 4.20 & 3.65 & 0.13 & 0.07 & Nil & Nil & 0.06 \\
\hline PN/W/109 & $\begin{array}{l}\text { Enyigba Mine } \\
\text { pond } 1\end{array}$ & Surface & 6.8 & 0.70 & 0.90 & 2.35 & 0.06 & 0.07 & Nil & 0.40 & 0.23 \\
\hline PN/W/110 & $\begin{array}{l}\text { Mine dump } \\
\text { drain, Enyigba }\end{array}$ & Surface & 6.6 & Nil & 3.70 & 1.10 & 0.09 & 0.02 & 0.08 & 0.04 & 0.06 \\
\hline PN/W/111 & $\begin{array}{l}\text { Enyigba Mine } \\
\text { pond } 2\end{array}$ & Surface & 6.3 & 0.40 & 1.10 & 0.20 & Nil & 0.05 & 3.63 & 0.10 & 0.16 \\
\hline PN/W/116 & $\begin{array}{l}\text { Ameri Mine } \\
\text { pond } 1\end{array}$ & Surface & 7.4 & 1.10 & 0.52 & 0.03 & 0.01 & 0.12 & Nil & 0.20 & 0.05 \\
\hline PN/W/117 & $\begin{array}{l}\text { Ameri Mine } \\
\text { pond } 2\end{array}$ & Surface & 6.0 & Nil & 3.00 & 0.00 & 0.14 & 3.46 & 0.57 & Nil & 0.74 \\
\hline PN/W/123 & $\begin{array}{l}\text { Ohankwu } \\
\text { Mine pond } 1\end{array}$ & Surface & 4.6 & Nil & Nil & Nil & 1.02 & 3.24 & Nil & 9.00 & 0.42 \\
\hline PN/W/124 & $\begin{array}{l}\text { Ohankwu } \\
\text { Mine pond } 2\end{array}$ & Surface & 3.9 & 1.40 & 11.00 & 2.90 & 0.91 & 1.80 & 1.89 & 5.70 & 0.95 \\
\hline $\mathrm{PN} / \mathrm{W} / 125$ & $\begin{array}{l}\text { Ohankwu } \\
\text { Mine pond } 3\end{array}$ & Surface & 5.1 & 2.20 & 3.00 & 4.19 & 0.04 & 0.18 & 2.23 & 7.50 & 0.32 \\
\hline $\mathrm{PN} / \mathrm{W} / 129$ & $\begin{array}{l}\text { Ameka Mine } \\
\text { pond } 1\end{array}$ & Surface & 4.2 & 0.30 & Nil & 1.20 & 1.22 & 0.06 & Nil & 1.40 & 1.66 \\
\hline PN/W/131 & $\begin{array}{l}\text { Ameka Mine } \\
\text { pond } 2\end{array}$ & Surface & 5.3 & Nil & 0.70 & Nil & 0.10 & 0.70 & 0.80 & 7.40 & 0.13 \\
\hline PN/W/132 & $\begin{array}{l}\text { Ameka Mine } \\
\text { pond } 3\end{array}$ & Surface & 3.4 & 2.40 & 9.10 & 0.19 & 0.06 & 1.28 & 0.38 & 5.00 & 0.32 \\
\hline PN/W/133 & $\begin{array}{l}\text { Ameka Mine } \\
\text { pond } 4\end{array}$ & Surface & 5.1 & Nil & 0.80 & Nil & 0.05 & 0.17 & Nil & 1.50 & 0.11 \\
\hline $\mathrm{PN} / \mathrm{W} / 115$ & $\begin{array}{l}\text { Ameri Mine } \\
\text { shaft }\end{array}$ & U/ground & 6.9 & 0.44 & 0.20 & 0.50 & 0.05 & 0.54 & 0.24 & 0.30 & 2.60 \\
\hline $\mathrm{PN} / \mathrm{W} / 120$ & $\begin{array}{l}\text { F. T. C. } \\
\text { Nwakpu, BH }\end{array}$ & U/ground & 6.2 & 0.01 & Nil & 2.40 & 0.16 & 0.08 & 0.31 & Nil & 7.50 \\
\hline PN/W/136 & $\begin{array}{l}\text { Ameka village } \\
\text { BH }\end{array}$ & U/ground & 5.5 & Nil & Nil & 0.97 & 0.13 & 0.08 & Nil & 0.10 & 0.27 \\
\hline $\mathrm{PN} / \mathrm{W} / 139$ & $\begin{array}{l}\text { Enyigba Prim. } \\
\text { Sch. BH }\end{array}$ & U/ground & 6.5 & 0.09 & 0.50 & 5.44 & 0.10 & 0.26 & Nil & Nil & 6.60 \\
\hline PN/W/145 & $\begin{array}{l}\text { Mine road, } \\
\text { Enyigba BH }\end{array}$ & U/ground & 6.5 & 0.13 & Nil & 2.40 & 0.09 & 0.41 & Nil & Nil & Nil \\
\hline TOTAL & & & 180.50 & 13.7 & 76.42 & 53.21 & 4.98 & 14.26 & 20.12 & 40.94 & 57.50 \\
\hline MEAN & & & 6.02 & 0.72 & 4.02 & 2.22 & 0.18 & 0.48 & 1.12 & 1.78 & 2.13 \\
\hline MAX & & & 7.40 & 2.50 & 13.50 & 5.44 & 1.22 & 3.46 & 3.63 & 9.00 & 10.10 \\
\hline MIN & & & 3.40 & 0.01 & 0.20 & 0.03 & 0.01 & 0.01 & 0.08 & 0.04 & 0.01 \\
\hline
\end{tabular}


Assessment of Contamination of Underground Water Sources in Enyigba Pb-Zn District, South Eastern Nigeria using Metal Enrichment and Pollution Indices

\begin{tabular}{|l|l|l|l|l|l|l|l|l|l|l|}
\hline \hline ST. DEV & & $\mathbf{1 . 0 1}$ & 0.79 & 4.19 & 1.63 & 0.31 & 0.86 & 0.89 & 2.81 & 3.24 \\
\hline $\begin{array}{l}\text { WHO (2011) Max. Limit } \\
\text { (mg/l) }\end{array}$ & & & .01 & .003 & NL & 2 & 0.4 & 0.07 & 0.01 & 3 \\
\hline
\end{tabular}

Exceeds the World Health Organization (WHO, 2011) Limit for Human

Consumption

Table 2 Concentration of heavy metals in underground water and its assessment with the maximum limits of World Health Organization (WHO, 2011). (No limit)

\begin{tabular}{|l|l|l|l|l|l|l|l|l|l|l|}
\hline $\begin{array}{l}\text { Sample } \\
\text { No }\end{array}$ & Location & $\mathbf{p H}$ & $\mathbf{A s}$ & $\mathbf{C d}$ & $\mathbf{C o}$ & $\mathbf{C u}$ & $\mathbf{M n}$ & $\mathbf{N i}$ & $\mathbf{P b}$ & $\mathbf{Z n}$ \\
\hline PN/W/120 & $\begin{array}{l}\text { F. T. C. } \\
\text { Nwakpu, BH }\end{array}$ & 6.2 & 0.01 & Nil & 2.40 & 0.16 & 0.08 & $\mathbf{0 . 3 1}$ & Nil & $\mathbf{7 . 5 0}$ \\
\hline PN/W/136 & $\begin{array}{l}\text { Ameka village } \\
\text { BH }\end{array}$ & 5.5 & Nil & Nil & 0.97 & 0.13 & 0.08 & Nil & $\mathbf{0 . 1 0}$ & 0.27 \\
\hline PN/W/139 & $\begin{array}{l}\text { Enyigba Prim. } \\
\text { Sch. BH }\end{array}$ & 6.5 & $\mathbf{0 . 0 9}$ & $\mathbf{0 . 5 0}$ & 5.44 & 0.10 & 0.26 & Nil & Nil & $\mathbf{6 . 6 0}$ \\
\hline PN/W/145 & $\begin{array}{l}\text { Mine road, } \\
\text { Enyigba BH }\end{array}$ & 6.5 & $\mathbf{0 . 1 3}$ & Nil & 2.40 & 0.09 & $\mathbf{0 . 4 1}$ & Nil & Nil & Nil \\
\hline TOTAL & & 24.70 & $\mathbf{0 . 2 3}$ & 0.50 & 11.21 & 0.48 & 0.83 & 0.31 & 0.10 & 14.37 \\
\hline MEAN & & 6.18 & 0.08 & 0.50 & 2.80 & 0.12 & 0.21 & 0.31 & 0.10 & 4.79 \\
\hline MAX & & 6.50 & 0.13 & 0.50 & 5.44 & 0.16 & 0.41 & 0.31 & 0.10 & 7.50 \\
\hline MIN & & 5.50 & 0.01 & 0.50 & 0.97 & 0.09 & 0.08 & 0.31 & 0.10 & 0.27 \\
\hline $\begin{array}{l}\text { STD. } \\
\text { DEV }\end{array}$ & 0.41 & 0.05 & 0.05 & 1.63 & 0.03 & 0.14 & 0.31 & 0.10 & 3.22 \\
\hline $\begin{array}{l}\text { WHO (2011) Max. Limit } \\
\text { (mg/l) }\end{array}$ & & .01 & .003 & NL & 2 & 0.4 & 0.07 & 0.01 & 3 \\
\hline
\end{tabular}

Exceeds the limits established by the World Health Organization (WHO, 2011)

Table 3 Maximum Limits of Metals Permissible in Water established by the World Health Organization (WHO, 2011).

\begin{tabular}{|c|c|}
\hline Parameter & Limits (mg/l) \\
\hline Arsenic & 0.01 \\
\hline Cadmium & 0.003 \\
\hline Copper & 2 \\
\hline Manganese & 0.4 \\
\hline Nickel & 0.07 \\
\hline Lead & 0.01 \\
\hline Zinc & 3 \\
\hline Cobalt & No limit established. \\
\hline
\end{tabular}

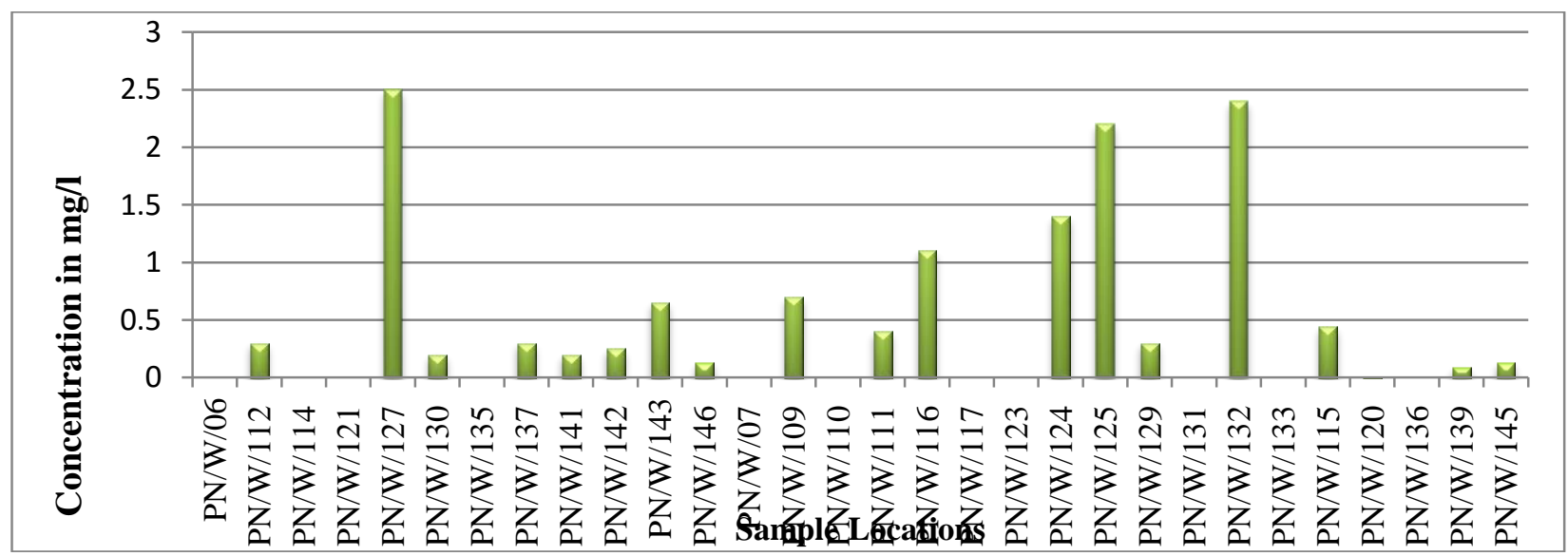

Figure 5 Concentration of arsenic in water from Enyigba and Environs 
High contents of As in water were recorded in a stream from Ohankwu area $(\mathrm{PN} / \mathrm{W} / 127)$ and one mine pond from Ameka (PN/W/132) and two mine ponds from Ohankwu (PN/W/125 and 124) areas with values of $2.5,2.4,2.2$ and $1.4 \mathrm{mg} / \mathrm{l}$ respectively (Tables 6 to 9; Figure 5).

$\mathrm{PN} / \mathrm{W} / 127$ is a stream that took its source from a mining hill at Ohankwu while PN/W/132, 125 and
124 are mine ponds where active local mining activities were taking place at the time the sampling took place. The content of $A s$ in the underground water was very low with a maximum value of 0.13 $\mathrm{mg} / \mathrm{l}$ obtained from a borehole at Enyigba and environs.

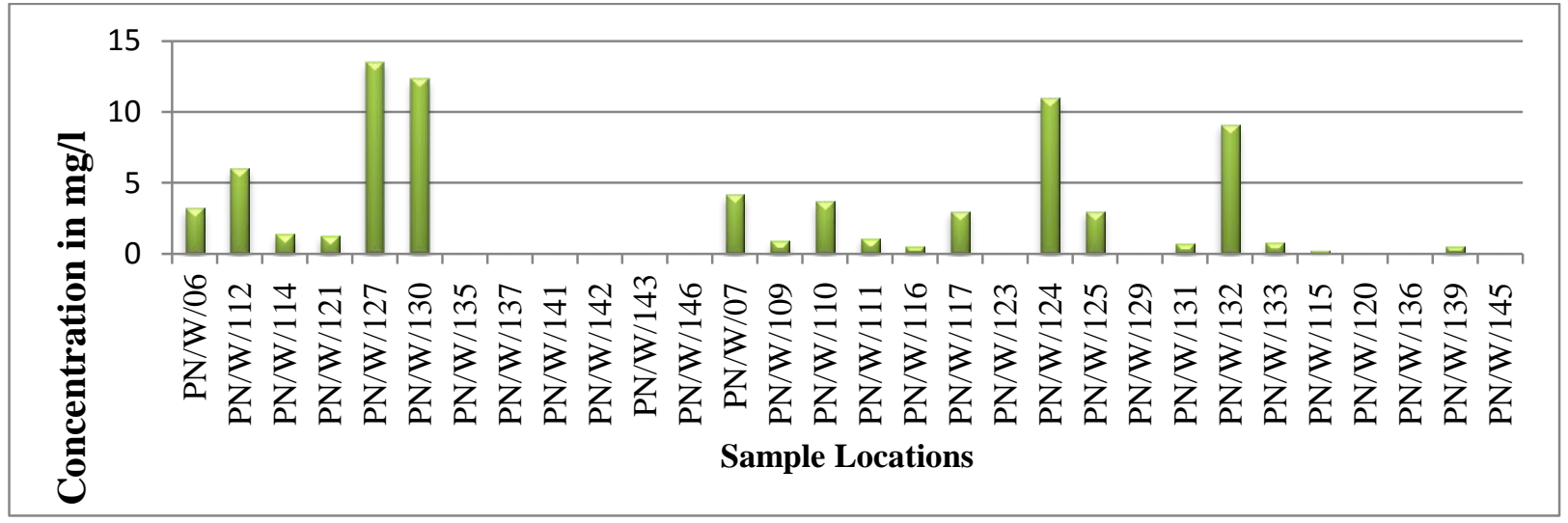

Figure 6 Concentration of cadmium in water from Enyigba and Environs

Very high concentration of $\mathrm{Cd}$ in water samples were recorded in two streams from Ohankwu (PN/W/127) and Ameka (PN/W/130) areas and two mine ponds from Ohankwu (PN/W/124) and Ameka (PN/W/132) areas with values of $13.5,12.3,11$ and $9.1 \mathrm{mg} / \mathrm{l}$ respectively (Tables 6 to 9; Figure 6). PN/W/127 and $\mathrm{PN} / \mathrm{W} / 130$ are streams that drain the mining areas at
Ohankwu and Ameka while PN/W/124 and $\mathrm{PN} / \mathrm{W} / 132$ are mine ponds where active local mining activities were taking place at the time the sampling took place. The content of $C d$ in the underground water was very low with a maximum value of 0.5 $\mathrm{mg} / \mathrm{l}$ obtained from a borehole at Enyigba Primary School (PN/W/139).

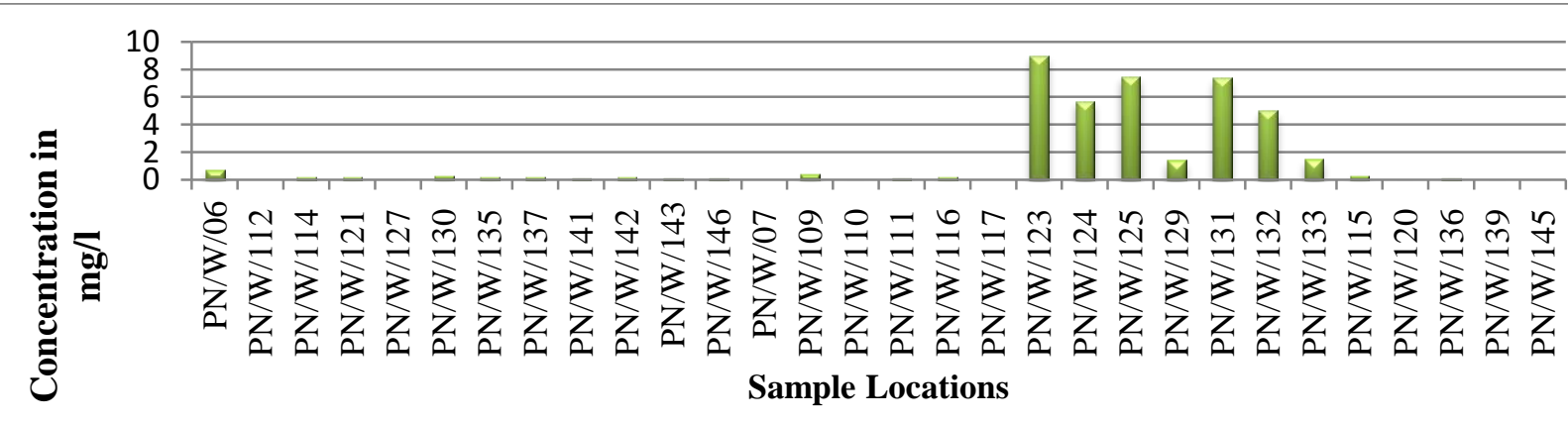

Figure 7 Concentration of lead in water from Enyigba and Environs

High values of $\mathrm{Pb}$ in water were concentrated in mine ponds from Ohankwu and Ameka areas (Tables 6 to 9; Figure 7). High concentrations of lead were recorded from samples $\mathrm{PN} / \mathrm{W} / 123, \mathrm{PN} / \mathrm{W} / 125$, $\mathrm{PN} / \mathrm{W} / 131, \mathrm{PN} / \mathrm{W} / 124$ and $\mathrm{PN} / \mathrm{W} / 132$ with values of $9,7.5,7.4,5.7$ and $5 \mathrm{mg} / \mathrm{l}$ respectively which are located within the $\mathrm{Pb}-\mathrm{Zn}$ mining areas. The contents of $\mathrm{Pb}$ in the surface and underground water samples were very low with a maximum value of $0.7 \mathrm{mg} / \mathrm{l}$ obtained from one stream at Enyigba and environs (PN/W/06).

\section{Metal Enrichment Index (MEI)}

Metal enrichment indices $(M E I)$ of heavy metals in water samples were calculated using the formula:

Metal Enrichment Index $M E I=\frac{c_{\tilde{\mathrm{i}}}-c_{0}}{c_{0}}$ 
Assessment of Contamination of Underground Water Sources in Enyigba $\mathrm{Pb}-\mathrm{Zn}$ District, South Eastern Nigeria using Metal Enrichment and Pollution Indices

Where $\mathrm{C}_{\mathrm{i}}$ is the total concentration of each metal $i$ measured in the water samples; $\mathrm{C}_{\mathrm{o}}$ is the heavy metal background established for the system studied.
The WHO maximum permissible limits of metals in water (see Table 3) were used as the background value for the metals.

Table 4 Metal enrichment indices of heavy metals in underground water.

\begin{tabular}{|l|l|l|l|l|l|l|l|l|l|}
\hline Sample No & Location & $\mathbf{p H}$ & $\mathbf{A s}$ & $\mathbf{C d}$ & $\mathbf{C u}$ & $\mathbf{M n}$ & $\mathbf{N i}$ & $\mathbf{P b}$ & $\mathbf{Z n}$ \\
\hline PN/W/120 & $\begin{array}{l}\text { F. T. C. } \\
\text { Nwakpu, BH }\end{array}$ & 6.2 & 0.01 & Nil & 0.16 & 0.08 & $\mathbf{0 . 3 1}$ & Nil & $\mathbf{7 . 5 0}$ \\
\hline PN/W/136 & $\begin{array}{l}\text { Ameka } \\
\text { village BH }\end{array}$ & 5.5 & Nil & Nil & 0.13 & 0.08 & Nil & $\mathbf{0 . 1 0}$ & 0.27 \\
\hline PN/W/139 & $\begin{array}{l}\text { Enyigba } \\
\text { Prim. Sch. } \\
\text { BH }\end{array}$ & 6.5 & $\mathbf{0 . 0 9}$ & $\mathbf{0 . 5 0}$ & 0.10 & 0.26 & Nil & Nil & $\mathbf{6 . 6 0}$ \\
\hline PN/W/145 & $\begin{array}{l}\text { Mine road, } \\
\text { Enyigba BH }\end{array}$ & 6.5 & $\mathbf{0 . 1 3}$ & Nil & 0.09 & $\mathbf{0 . 4 1}$ & Nil & Nil & Nil \\
\hline TOTAL & & & $\mathbf{0 . 2 3}$ & 0.50 & 0.48 & 0.83 & 0.31 & 0.10 & 14.37 \\
\hline MEI & & & 22 & 165.67 & -0.76 & 1.08 & 3.43 & 9 & 3.79 \\
\hline WHO (2008) Max. Limit (mg/l) & $\mathbf{0 . 0 1}$ & $\mathbf{0 . 0 0 3}$ & $\mathbf{2}$ & $\mathbf{0 . 4}$ & $\mathbf{0 . 0 7}$ & $\mathbf{0 . 0 1}$ & $\mathbf{3}$ \\
\hline
\end{tabular}

Exceeds the limits established by the World Health Organization (WHO, 2011)

Table 4 and Figure 8 show that in the underground water samples, Cd had the highest metal enrichment value of 165.67 followed by $\mathrm{As}, \mathrm{Pb}, \mathrm{Zn}$ and $\mathrm{Ni}$ with values of 22, 9, 3.79 and 3.43 respectively.

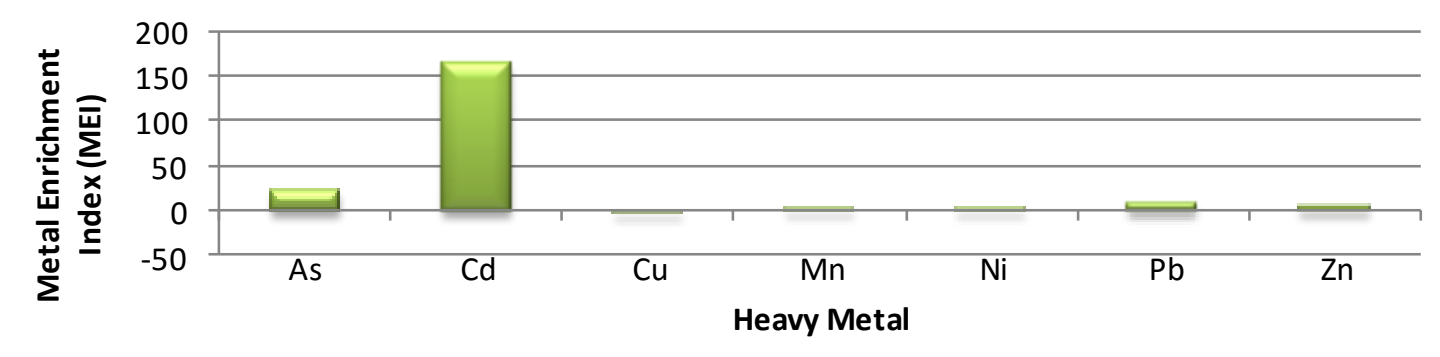

Figure 8 Metal enrichment index of heavy metals in underground water samples

Metal Pollution Index (MPI).

Metal pollution index (MPI) is calculated using the formula (Caeiro et al 2005):

$$
M P I=\sqrt[\mathrm{n}]{\mathrm{M} 1 \mathrm{XM} 2 \mathrm{XM} \text { M X ......Mn }}
$$

Where $\mathrm{M} n$ is the concentration of metal $n$ in the water samples expressed in $\mathrm{mg} / \mathrm{l}$. This is simple but does not compare the contaminant concentration with any baseline value. The calculated metal pollution index of the heavy metals in the water samples of the area are shown on Table 5 and plotted in Figure 9.

Table 5 Metal Pollution Index $(M P I)$ of heavy metals in Water from Enyigba and Environs

\begin{tabular}{|l|l|l|l|l|l|l|l|l|l|l|l|l|}
\hline $\begin{array}{l}\text { Sample } \\
\text { No }\end{array}$ & Location & $\begin{array}{l}\text { Sample } \\
\text { type }\end{array}$ & $\mathbf{P h}$ & $\mathbf{A s}$ & $\mathbf{C d}$ & $\mathbf{C o}$ & $\mathbf{C u}$ & $\mathbf{M n}$ & $\mathbf{N i}$ & $\mathbf{P b}$ & $\mathbf{Z n}$ & $\mathbf{M P I}$ \\
\hline PN/W/06 & $\begin{array}{l}\text { Enyigba } \\
\text { Stream 1 }\end{array}$ & Surface & 7.0 & Nil & 3.2 & 4.2 & 0.17 & 0.42 & 1.49 & 0.7 & 0.56 & $\mathbf{0 . 9 2}$ \\
\hline PN/W/112 & $\begin{array}{l}\text { Enyigba } \\
\text { stream 2 }\end{array}$ & Surface & 6.6 & 0.3 & 6.0 & 1.26 & 0.08 & 0.06 & 1.43 & Nil & 0.54 & $\mathbf{0 . 5 2}$ \\
\hline PN/W/114 & Ameri stream & Surface & 6.7 & Nil & 1.40 & 1.71 & 0.06 & 0.11 & Nil & 0.2 & 0.09 & $\mathbf{0 . 2 6}$ \\
\hline PN/W/121 & $\begin{array}{l}\text { Ohankwu } \\
\text { stream 1 }\end{array}$ & Surface & 6.2 & Nil & 1.30 & 0.60 & 0.05 & 0.02 & 0.88 & 0.20 & 0.01 & $\mathbf{0 . 1 5}$ \\
\hline PN/W/127 & $\begin{array}{l}\text { Ohankwu } \\
\text { stream 2 }\end{array}$ & Surface & 5.5 & 2.50 & 13.5 & Nil & 0.02 & 0.21 & 0.97 & Nil & Nil & $\mathbf{0 . 6 7}$ \\
\hline PN/W/130 & $\begin{array}{l}\text { Ameka stream } \\
1\end{array}$ & Surface & 5.8 & 0.20 & 12.3 & Nil & Nil & 0.07 & 0.42 & 0.30 & 0.15 & $\mathbf{0 . 3 8}$ \\
\hline PN/W/135 & Ameka stream & Surface & 5.6 & Nil & Nil & 0.97 & 0.02 & 0.04 & Nil & 0.20 & 0.27 & $\mathbf{0 . 1 3}$ \\
\hline
\end{tabular}


Assessment of Contamination of Underground Water Sources in Enyigba Pb-Zn District, South Eastern Nigeria using Metal Enrichment and Pollution Indices

\begin{tabular}{|c|c|c|c|c|c|c|c|c|c|c|c|c|}
\hline & 2 & & & & & & & & & & & \\
\hline $\mathrm{PN} / \mathrm{W} / 137$ & $\begin{array}{l}\text { Enyigba villa } \\
\text { pond }\end{array}$ & Surface & 6.0 & 0.30 & Nil & 4.60 & 0.06 & 0.20 & 2.07 & 0.20 & Nil & 0.46 \\
\hline $\mathrm{PN} / \mathrm{W} / 141$ & $\begin{array}{l}\text { Enyigba villa } \\
\text { stream } 1\end{array}$ & Surface & 6.8 & 0.20 & Nil & 2.39 & 0.03 & 0.26 & 1.58 & 0.10 & 9.70 & 0.52 \\
\hline $\mathrm{PN} / \mathrm{W} / 142$ & $\begin{array}{l}\text { Ameka villa } \\
\text { stream }\end{array}$ & Surface & 6.6 & 0.25 & Nil & 5.06 & 0.08 & 0.01 & 0.52 & 0.20 & 6.40 & 0.35 \\
\hline $\mathrm{PN} / \mathrm{W} / 143$ & $\begin{array}{l}\text { Ebonyi River, } \\
\text { Enyigba }\end{array}$ & Surface & 7.0 & 0.65 & Nil & 3.90 & 0.04 & 0.197 & Nil & 0.10 & 10.1 & 0.52 \\
\hline $\mathrm{PN} / \mathrm{W} / 146$ & $\begin{array}{l}\text { Enyigba villa } \\
\text { stream } 2\end{array}$ & Surface & 7.2 & 0.13 & Nil & 1.0 & 0.01 & 0.07 & 0.63 & 0.10 & 7.50 & 0.19 \\
\hline PN/W/07 & $\begin{array}{l}\text { Enyigba Shale } \\
\text { quarry pond }\end{array}$ & Surface & 7.2 & Nil & 4.20 & 3.65 & 0.13 & 0.07 & Nil & Nil & 0.06 & 0.40 \\
\hline PN/W/109 & $\begin{array}{l}\text { Enyigba Mine } \\
\text { pond } 1\end{array}$ & Surface & 6.8 & 0.70 & 0.90 & 2.35 & 0.06 & 0.07 & Nil & 0.40 & 0.23 & 0.34 \\
\hline $\mathrm{PN} / \mathrm{W} / 110$ & $\begin{array}{l}\text { Mine dump } \\
\text { drain, Enyigba }\end{array}$ & Surface & 6.6 & Nil & 3.70 & 1.10 & 0.09 & 0.02 & 0.08 & 0.04 & 0.06 & 0.15 \\
\hline $\mathrm{PN} / \mathrm{W} / 111$ & $\begin{array}{l}\text { Enyigba Mine } \\
\text { pond } 2\end{array}$ & Surface & 6.3 & 0.40 & 1.10 & 0.20 & Nil & 0.05 & 3.63 & 0.10 & 0.16 & 0.31 \\
\hline PN/W/116 & $\begin{array}{l}\text { Ameri Mine } \\
\text { pond } 1\end{array}$ & Surface & 7.4 & 1.10 & 0.52 & 0.03 & 0.01 & 0.12 & Nil & 0.20 & 0.05 & 0.11 \\
\hline PN/W/117 & $\begin{array}{l}\text { Ameri Mine } \\
\text { pond } 2\end{array}$ & Surface & 6.0 & Nil & 3.00 & 0.00 & 0.14 & 3.46 & 0.57 & Nil & 0.74 & 0.91 \\
\hline $\mathrm{PN} / \mathrm{W} / 123$ & $\begin{array}{l}\text { Ohankwu Mine } \\
\text { pond } 1\end{array}$ & Surface & 4.6 & Nil & Nil & Nil & 1.02 & 3.24 & Nil & 9.00 & 0.42 & 1.88 \\
\hline PN/W/124 & $\begin{array}{l}\text { Ohankwu Mine } \\
\text { pond } 2\end{array}$ & Surface & 3.9 & 1.40 & 11.00 & 2.90 & 0.91 & 1.80 & 1.89 & 5.70 & 0.95 & 2.29 \\
\hline $\mathrm{PN} / \mathrm{W} / 125$ & $\begin{array}{l}\text { Ohankwu Mine } \\
\text { pond } 3\end{array}$ & Surface & 5.1 & 2.20 & 3.00 & 4.19 & 0.04 & 0.18 & 2.23 & 7.50 & 0.32 & 1.01 \\
\hline PN/W/129 & $\begin{array}{l}\text { Ameka Mine } \\
\text { pond } 1\end{array}$ & Surface & 4.2 & 0.30 & Nil & 1.20 & 1.22 & 0.06 & Nil & 1.40 & 1.66 & 0.63 \\
\hline PN/W/131 & $\begin{array}{l}\text { Ameka Mine } \\
\text { pond } 2\end{array}$ & Surface & 5.3 & Nil & 0.70 & Nil & 0.10 & 0.70 & 0.80 & 7.40 & 0.13 & 0.58 \\
\hline PN/W/132 & $\begin{array}{l}\text { Ameka Mine } \\
\text { pond } 3\end{array}$ & Surface & 3.4 & 2.40 & 9.10 & 0.19 & 0.06 & 1.28 & 0.38 & 5.00 & 0.32 & 0.81 \\
\hline PN/W/133 & $\begin{array}{l}\text { Ameka Mine } \\
\text { pond } 4\end{array}$ & Surface & 5.1 & Nil & 0.80 & Nil & 0.05 & 0.17 & Nil & 1.50 & 0.11 & 0.26 \\
\hline PN/W/115 & $\begin{array}{l}\text { Ameri Mine } \\
\text { shaft }\end{array}$ & U/ground & 6.9 & 0.44 & 0.20 & 0.50 & 0.05 & 0.54 & 0.24 & 0.30 & 2.60 & 0.35 \\
\hline $\mathrm{PN} / \mathrm{W} / 120$ & $\begin{array}{l}\text { F. T. C. } \\
\text { Nwakpu, } \\
\text { Borehole }\end{array}$ & U/ground & 6.2 & 0.01 & Nil & 2.40 & 0.16 & 0.08 & 0.31 & Nil & 7.50 & 0.30 \\
\hline PN/W/136 & $\begin{array}{l}\text { Ameka village } \\
\text { Borehole }\end{array}$ & U/ground & 5.5 & Nil & Nil & 0.97 & 0.13 & 0.08 & Nil & 0.10 & 0.27 & 0.19 \\
\hline PN/W/139 & $\begin{array}{l}\text { Enyigba Prim. } \\
\text { Sch. Borehole }\end{array}$ & U/ground & 6.5 & 0.09 & 0.50 & 5.44 & 0.10 & 0.26 & Nil & Nil & 6.60 & 0.58 \\
\hline $\mathrm{PN} / \mathrm{W} / 145$ & $\begin{array}{l}\text { Mine road, } \\
\text { Enyigba } \\
\text { Borehole }\end{array}$ & U/ground & 6.5 & 0.13 & Nil & 2.40 & 0.09 & 0.41 & Nil & Nil & Nil & 0.32 \\
\hline$\leq 1$ & $1-5$ & $5-2$ & & & -40 & & $>$ & 40 & & & & \\
\hline $\begin{array}{l}\text { Practical } \\
\text { unpollute }\end{array}$ & $\begin{array}{l}\text { Slightly } \\
\text { polluted }\end{array}$ & $\begin{array}{r}\text { Modera } \\
\text { pollute }\end{array}$ & & & $\begin{array}{l}\text { ongly } \\
\text { luted }\end{array}$ & & $\begin{array}{l}\text { stro } \\
\text { llute }\end{array}$ & & & & & \\
\hline
\end{tabular}

It was only three mine ponds that had slight pollution based on calculated metal pollution index values. The highest value of 2.29 was recorded in location PN/W/124 (Table 5; Figure 9). 


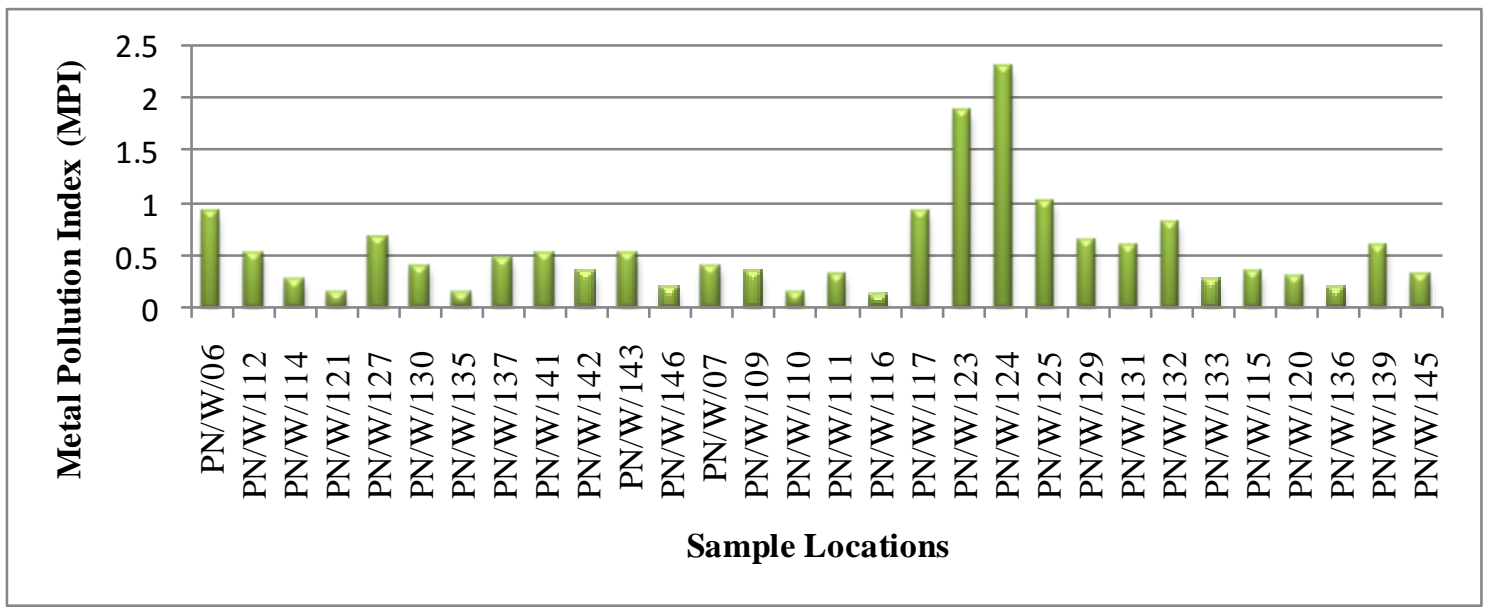

Figure 9 Metal Pollution Index of heavy metals in Water from Enyigba and Environs

\section{DISCUSSION}

In underground water samples, high content of As was recorded from boreholes at Mine Road, Enyigba and Enyigba Primary School with concentrations of 0.13 and $0.09 \mathrm{mg} / \mathrm{l}$ respectively. These values are 13 and 9 times above the WHO (2011) maximum permissible limit of $0.01 \mathrm{mg} / \mathrm{l}$ for water quality (Table 4, Figure 5). The average content of $0.08 \mathrm{mg} / \mathrm{l}$ of As in underground water is 8 times above the WHO (2011) limit for water quality (Table 5). These concentrations make the water unsafe for human consumption.

High content of Cd was only obtained from Enyigba Primary School borehole with concentration of 0.5 $\mathrm{mg} / \mathrm{l}$, and 167 times above the WHO (2011) maximum permissible limit of $0.003 \mathrm{mg} / \mathrm{l}$ for water quality (Table 4, Figure 6). The average content of $0.5 \mathrm{mg} / \mathrm{l}$ of $\mathrm{Cd}$ in underground water is also 167 times above the WHO (2011) limit for water quality (Table 5). This concentration renders this water source risky for human consumption.

High content of $\mathrm{Pb}$ was only obtained from a borehole at Ameka village with concentration of 0.1 $\mathrm{mg} / \mathrm{l}$, and 10 times above the WHO (2011) maximum permissible limit of $0.01 \mathrm{mg} / \mathrm{l}$ for water quality (Table 4, Figure 7). The average content of $0.1 \mathrm{mg} / \mathrm{l}$ of $\mathrm{Pb}$ in underground water is only 10 times above the WHO (2011) limit for water quality (Table 4). This is expected as local $\mathrm{Pb} / \mathrm{Zn}$ mining is very active in the area. Cidu and Fanfani (2000)'s investigation of the impact of abandoned mines on water resources in SW Sardinia area (Italy) showed that pore water in dumps exhibited high contents of $\mathrm{Pb}, \mathrm{Cu}, \mathrm{Co}, \mathrm{Ni}, \mathrm{Cd}$ and $\mathrm{Zn}$. This was also obtainable in Humboldt $\mathrm{Pb}-\mathrm{Zn}-$ $\mathrm{Cu}$ mines (N. Nevada) where the water sources were polluted by $\mathrm{As}, \mathrm{Cd}, \mathrm{Cu}, \mathrm{Mn}, \mathrm{Pb}$ and $\mathrm{Zn}$ from the mine dumps (Nash, 2003). However, the abundance of siderite and other carbonate minerals in Sardinia area produced a near neutral $\mathrm{pH}$ in the streams.

High content of $\mathrm{Ni}$ was only obtained from a borehole at Folk Technical College, Nwakpu, Ikwo with concentration of $0.31 \mathrm{mg} / \mathrm{l}$, and only 4 times above the WHO (2011) maximum permissible limit of $0.07 \mathrm{mg} / \mathrm{l}$ for water quality (Table 4 ). The average content of $0.31 \mathrm{mg} / \mathrm{l}$ of $\mathrm{Ni}$ in underground water is only 4 times above the WHO (2011) limit for water quality and little contribution to the overall pollution index of metals in underground water. This is because the larger part of all $\mathrm{Ni}$ compounds that are released to the environment will adsorb to sediments or soil particles and become immobile as a result. It becomes more mobile and enters into groundwater only in acidic environments.

High content of $\mathrm{Zn}$ was only obtained from boreholes at Folk Technical College, Nwakpu, Ikwo and Enyigba Primary School with concentrations of 7.5 and $6.6 \mathrm{mg} / \mathrm{l}$ respectively, which are 2.5 and 2.2 times above the WHO (2011) maximum permissible limit of $3 \mathrm{mg} / \mathrm{l}$ for water quality (Table 4). This may be explained by the possibility of deep-seated $\mathrm{Pb} / \mathrm{Zn}$ vein in these areas. The average content of $4.79 \mathrm{mg} / \mathrm{l}$ of $\mathrm{Zn}$ in underground water is only 1.6 times above the WHO (2011) limit for water quality (Table 4). Water-soluble $\mathrm{Zn}$ in soils can contaminate groundwater. However, $\mathrm{Zn}$, if in solution phase in soil would be highly attenuated through adsorption reactions thereby reducing its mobility (Mattigod and Page, 1983).

The distribution of cobalt in water from the Enyigba and Environs ranged from 0.03 to $5.44 \mathrm{mg} / \mathrm{l}$ with an average value of $2.22 \pm 1.63 \mathrm{mg} / \mathrm{l}$ with the highest value of $5.44 \mathrm{mg} / \mathrm{l}$ recorded from a borehole at Enyigba Primary School. Lower values were obtained from boreholes at Folk Technical College, 
Nwakpu, Ikwo and Mine Road, Enyigba with concentrations of $2.4 \mathrm{mg} / \mathrm{l}$. Other high values were obtained from streams and surface pond at Ameka village and Enyigba with values of 4.6 and $4.2 \mathrm{mg} / \mathrm{l}$ respectively (Tables 6 to 9). The highest content of $\mathrm{Co}$ in mine ponds was from Ohankwu and Enyigba areas. Elevated concentrations of Co were obtained from Ebonyi River, and streams and shale quarry pond at Enyigba.

The only high content of Mn was from a borehole from Mine Road, Enyigba with a concentration of $0.41 \mathrm{mg} / \mathrm{l}$, which is only 1.03 times the WHO (2011) maximum permissible limit $(0.4 \mathrm{mg} / \mathrm{l})$ for water quality (Table 4). Pollution from mining activities is particularly difficult to deal with because it lasts for decades after mine closure (Bolucek, 2007) but monitoring the metals in water is important for safety assessment of the environment and human health in particular (Kar et al. 2008). In Trezebionka (Poland), where intensive mining activities took place, the mines and ground waters were reported to be characterized by higher contents of heavy metals including $\mathrm{Cd}, \mathrm{Cu}, \mathrm{Co}, \mathrm{Ni}, \mathrm{Pb}, \mathrm{Zn}$ and $\mathrm{Mn}$. These polluting heavy metals originated from mining and extraction activities in the area (Gajowiec and Witkowski, 1993). The groundwater was naturally enriched in heavy metals but previous mining activities resulted in highly polluted and toxic groundwater at the vicinity of the mines by $\mathrm{Pb}, \mathrm{Cu}$, $\mathrm{Cd}$ and $\mathrm{Mn}$. In the historical mining areas of Montana and Colorado, ore-related metal concentrations in many stream reaches draining the mined areas have elevated concentrations of $\mathrm{Cd}, \mathrm{Pb}, \mathrm{As}, \mathrm{Cu}$ and $\mathrm{Zn}$ that normally exceed the allowable levels for aquatic life (Nimick et al. 1999).

\section{Metal Enrichment Index (MEI) and Metal Pollution Index (MPI)}

The metal enrichment index of As in the water from the area was high in surface water, in mine ponds and in underground water (Figure 8), and all indicated high contamination but more in mine ponds followed by surface water.

The metal enrichment index of $\mathrm{Cd}$ in the water samples were high varying from 12566 in surface water 12739 in mine ponds and down to 165.67 in underground water (Figure 8), and all indicating high contamination but more in surface water and mine ponds followed by the underground water. As and $\mathrm{Cd}$ were possibly adsorbed on to soil particles.

The metal enrichment index of $\mathrm{Ni}$ in the water samples was high varying from 141.7 in surface water, 139 in mine ponds and down to 3.43 in underground water (Figure 8), indicating high contamination in surface water and mine ponds and moderate pollution in underground water. This may be due to low very low mobility of $\mathrm{Ni}$ in neutral to alkaline conditions (Plant and Raiswell, 1983). The metal enrichment index of $\mathrm{Cu}$ in mine pond water samples was very low and negative in surface and underground water (Figures 8).

The metal enrichment index of $\mathrm{Pb}$ in the water samples was high varying from 229 in surface water to 3853 in mine ponds and down to 9 in underground water (Figure 8) and all indicating high contamination but more in mine ponds followed by surface water and underground water. This may be due to low very low mobility of $\mathrm{Pb}$ in neutral to alkaline conditions (Plant and Raiswell, 1983).

The metal enrichment index of $\mathrm{Zn}$ in surface and mine pond water was very low and 70) but attained a value of 3.79 in underground water (Figure 8), which indicated moderate pollution in underground water and low to no pollution in surface and mine ponds water. The metal enrichment index of $\mathrm{Mn}$ in all the water samples was very low (Figures 8 ).

\section{CONCLUSION}

The MEI of As in water was very high: highest (893) in mine ponds, followed by surface water (452) and lowest in underground water (22), all indicating high contamination by As. Based on PLI, As contributed to very high pollution of surface water in Ohankwu and Ameka; mine ponds in Enyigba, Ohankwu and Ameka; high pollution in mine ponds Ameka, Ohankwu and Ameri, and moderate pollution in Enyigba Primary School borehole, and Ameri. Based on MPI, As made significant contribution to the slight pollution in Ohankwu mine ponds.

The MEI of $\mathrm{Cd}$ in water was high and varying from 12739 in mine ponds, 12566 in surface water down to 165 in underground water, all indicating high degree of contamination. Based on PLI, Cd contributed to very high pollution of surface water in Ohankwu and Ameka; mine ponds in Ohankwu, Ameka and Enyigba; high pollution in surface water in Enyigba; mine ponds Ameka and Ameri, and moderate pollution in Ameri, and Enyigba Primary School borehol. Based on MPI, Cd made significant contribution to the slight pollution in Ohankwu mine ponds.

The MEI of $\mathrm{Pb}$ in water was high and varying from 3853 in mine ponds, 229 in surface water down to 9 in underground water, and all indicating varying degrees high contamination. The high level of contamination expressed by the concentrations of As, $\mathrm{Cd}$ and $\mathrm{Pb}$ were related to the $\mathrm{Pb} / \mathrm{Zn}$ occurrence and mining in the study area. This is also shown by the positive correlation between $\mathrm{Pb}, \mathrm{Cu}$ and $\mathrm{Zn}$ which 
indicates the effects of man's activities on the source environment of the metals. Based on PLI, Pb made contribution to very high pollution of surface water in Ameka; mine ponds in Ohankwu, Ameka and Enyigba; high pollution of surface water in Enyigba, mine ponds in Ohankwu and Ameka. Based on MPI, $\mathrm{Pb}$ made very significant contribution to the slight pollution in Ohankwu ponds.

The MEI of Ni in water was high, varying from 142 in surface water, 139 in in mine ponds and 3.43 in underground water, indicating high contamination in surface and mine pond water, and moderate contamination in underground water. Based on PLI, $\mathrm{Ni}$ contributed to very high pollution of surface water in Ohankwu and Ameka; mine ponds in Enyigba, Ohankwu and Ameka; high pollution of surface water in Enyigba, Ameri and Ameka. It also contributed significantly to the slight pollution in Ohankwu mine ponds based on MPI.

\section{REFERENCE}

1) Agumanu, A. E. (1989). The Abakaliki and Ebonyi Formations: subdivisions of the Albian Asu River Group in the the southern Benue trough, Nigeria. Journal of African Earth Sciences.Vol. 9, No 1, pp. 195-207.

2) Bölücek, C. (2007). Environmental contamination in the Keban mining district, eastern Turkey. Arabian Journal of Science and Engineering 32 (1A), 3-18.

3) Caeiro, S., Costa, M. H., Ramos, T. B., Fernandes, F., Silveira, N., Coimbra, A., Medeiros, G. and Painho, M. (2005). Assessing heavy metal contamination in Sado estuary sediment: an index analysis approach. Ecological Indicators 5, 151-169.

4) Cidu, R. and Fanfani, L. (2000). Impact of dismantled mines on water resources in Sardinia (Italy). Retrieved from www.cprm.gov.br/pgagcm/Manuscripts/cidur.htm.on 30/05/2011

5) Farrington, J. L. (1952). A Preliminary Description of the Nigerian leadzinc field. Econ. Geol 47 (6), 583-608.

6) Gajowiec, B. and Witkowski, A. (1993). Impact of lead/zinc mining on groundwater quality in Trzebionka mine (Southern Poland), Mine Water and Environment 12, 1-10

7) Hoque, M. (1977). Petrographic Differentiation of Tectonically Controlled Cretaceous Sedimentary Cycles, Southeast Nigeria, Sed. Geol., 17, 235245.
8) Igwe, O., Adepehin, E. I. and Iwuanyanwu, C. (2012). Environmental effects of the mining of lead-zinc minerals in Enyigba and its suburbs, southern Bebue Trough, Nigeria. Nigeria Journal of Education, Heath and Technology Research, 3 (2): 30-44.

9) Kogbe, C. A. (1989). The Cretaceous and Paleogene sediments of Southern Nigeria, In Geology of Nigeria, C. A. Kogbe (ed). Rock View Nigeria Limited, Jos. pp. 325-334

10) Mattigod, S. V. and Page, A. L. (1983). Assessment of metal pollution in soils. In: Thornton, I. (ed) Applied Environmental Geochemistry, Academic Press, London, 355-393.

11) Nash, J. T. (2003). Historic mills and mill tailings as potential sources of contamination in and near the Humboldt River Basin, Northern Nevada, USGS Bulletin 2210 - D, 36p.

12) Nimick, D. A., Church, S. E., Cleasby, T. E., Fey, D. L., Kimball, B. A., Leib, K. J., Mast, M. A. and Wright, W. G. (1999). Characterization of metals in water and bed sediment in two watersheds affected by historical mining in Montana and Colorado. Retrieved from: http://toxics.usgs.gov/pubs/wri99-4018/vol1/sectionA/1201Nimick/pdf/Nimick.pdf. on 03/04/2011.

13) Nnabo, P. N. (2011). Environmental impacts of lead/zinc mining in Enyigba area, SE of Abakaliki, SE Nigeria. Ph.D Thesis, Ebonyi State University, Abakaliki, Nigeria. 322p.

14) Nnabo, P. N. (2015). Assessment of heavy metal distribution in rocks from Enyigba $\mathrm{Pb}-\mathrm{Zn}$ district, southeastern Nigeria. International Journal of Innovation and Scientific Research, 17 (1): 175-185.

15) Nnabo, P. N., Orazulike, D. M. and Offor, C. O. (2011). The preliminary assessment of the level of heavy Elements contaminations in stream bed sediments of Enyigba and environs, south eastern Nigeria Journal of Basic Physical Research, Vol. 2, No.2, pp. $43-52$.

16) Onyeobi, T. U. S. and Imeokparia, E. G. (2014). Heavy metal contamination and distribution in soils around $\mathrm{Pb}-\mathrm{Zn}$ mines of Abakaliki district, southeastern Nigeria. Frontiers in Geosciences, 2 (2): 30-40.

17) Orajaka, S. (1965). The Geology of Enyigba, Ameri and Ameka Lead-Zinc lodes Abakaliki; South-eastern Nigeria. A reconnaissance. J. Min. Geol. 2, 65-69.

18) Orazulike, D. M. (1994). The mineralogy and texture of lead - zinc copper ores of Enyigba lode, Abakaliki, Nigeria, Journal of Mining and Geology 30 (1), 25-32.

19) Plant, J. A. and Raiswell, R. (1983). Principles of environmenta geochemistry. In: Thornton, I. (ed) Applied Environmental Geochemistry, Academic Press, London, 1-39.

20) World Health Organization (2011). Guidelines for drinking-water quality [electronic resource]: incorporating 1st and 2nd addenda. Vol. 1, Recommendations, 5 th edition. Geneva. $668 \mathrm{p}$ 